\title{
Immunoglobulin (Ig) G4-related sclerosing cholangitis in patients resected for presumed perihilar cholangiocarcinoma: a 10-year experience
}

\author{
Lei Feng $^{1 \# \wedge}$, Zhen You ${ }^{1 \#}$, Dongyang Ma ${ }^{1 \#}$, Lvjun Yan $^{2 \#}$, Hua Cheng ${ }^{3 \#}$, Junhe Gou $^{4}$, Liping Chen ${ }^{1}$ \\ ${ }^{1}$ Department of Biliary Surgery, West China Hospital, Sichuan University, Chengdu, China; ${ }^{2}$ Department of Oncology, Second Affiliated Hospital \\ of Army Medical University, Chongqing, China; ${ }^{3}$ Department of Intensive Care Unit, West China Hospital, Sichuan University, Chengdu, China; \\ ${ }^{4}$ Department of Pathology, West China Hospital, Sichuan University, Chengdu, China \\ Contributions: (I) Conception and design: L Feng, Z You; (II) Administrative support: L Chen; (III) Provision of study materials or patients: L Feng, \\ J Gou; (IV) Collection and assembly of data: L Feng, D Ma, L Yan; (V) Data analysis and interpretation: L Feng, L Yan, H Cheng; (VI) Manuscript \\ writing: All authors; (VII) Final approval of manuscript: All authors. \\ \#These authors contributed equally to this work. \\ Correspondence to: Liping Chen. Department of Biliary Surgery, West China Hospital, Sichuan University, No. 37 of Guoxue Alley, Wuhou District, \\ Chengdu 610041, China. Email: 47137417@qq.com.
}

Background: This study aimed to investigate the incidence of immunoglobulin (Ig) G4-related sclerosing cholangitis (IgG4-SC) in patients resected for perihilar cholangiocarcinoma (PHC) in a designated hospital from 2010 to 2019. We also aimed to evaluate the diagnostic dilemma of IgG4-SC clinically.

Methods: Between January 2010 and December 2019, all patients who underwent radical resection due to presumed PHC were included. Independent pathologists scored bile duct samples based on the International Consensus Pathology Criteria for IgG4-related Disease (ICPD).

Results: Of the 289 patients who underwent radical resection of primary liver cancer, 26 (9\%) were diagnosed as benign, without histological evidence of malignancy, among them, 23 had sclerosing inflammation, 1 had cystadenoma, and 2 had xanthogranulomatous cholangitis. Additionally, 18 had a definitive diagnosis of IgG4-SC. The misdiagnosis rate was 19\% (54/289), of which, 26 patients had benign disease, and 28 patients had malignancies.

Conclusions: It is difficult to distinguish IgG-SC from PHC. The misdiagnosis has resulted in a large number of ineffective hepatectomies. Improving the detection rate of serum IgG4 (sIgG4) may therefore avoid misdiagnosis, surgery, and life-threatening complications.

Keywords: Immunoglobulin (Ig) G4-related sclerosing cholangitis; perihilar cholangiocarcinoma; misdiagnosis; extensive surgery; retrospective study

Submitted Oct 08, 2020. Accepted for publication Jan 29, 2021.

doi: $10.21037 /$ atm-21-140

View this article at: http://dx.doi.org/10.21037/atm-21-140

\section{Introduction}

Immunoglobulin (Ig) G4-related sclerosing cholangitis (IgG4-SC), also called IgG4-associated cholangitis (IAC) (1), is a multi-organ inflammatory disorder which is responsive to steroid therapy (2). Distinguishing IgG4-SC from perihilar cholangiocarcinoma (PHC) is challenging as the presentation of these diseases may be similar, and accurate diagnostic serological markers

\footnotetext{
$\wedge$ ORCID: 0000-0003-4361-2771.
} 
have still not been found. Therefore, up to one-third of patients, before being diagnosed with IgG4-SC, underwent surgery that was presumed to be for PHC (3). The general diagnostic methods for IgG4-SC include preoperative imaging observations, endoscopic retrograde cholangiopancreatography (ERCP), brush cytology, and detection of sIgG4. Imaging examinations of IgG4-SC can detect the extrahepatic bile duct or the bile duct around the porta hepatis, which is one of the signs of primary liver cancer.

In a series of reports prior to 2008, the benign lesion resection rate of $\mathrm{PHC}$ was considered to vary from $8 \%$ to $22 \%$ (4). It is unclear whether this was due to IgG4SC. The International Consensus Pathology Criteria for IgG4-related Disease (ICPD) have been developed to determine the diagnosis of IgG4-SC in resected specimens (5). Pathological sections are characterized by infiltration of lymphocytes and a large number of IgG4 plasma cells, accompanied by typical histopathological changes, including storage fibrosis and obliterative phlebitis.

The purpose of this study was to investigate the incidence rate of IgG4-SC in patients with primary liver cancer and to reveal whether the incidence rate of IgG4SC has declined in the 10 years since 2010. In addition, we aimed to evaluate the diagnostic dilemma of IgG4-SC.

We present the following article in accordance with the STROBE reporting checklist (available at http://dx.doi. org/10.21037/atm-21-140).

\section{Methods}

\section{Study subjects and data collection}

All patients who underwent radical resection of presumed primary liver cancer in West China Hospital of Sichuan University from January 2010 to December 2019 were included. Demographic characteristics, initial symptoms, clinical manifestations (jaundice, abdominal pain, weight loss, nausea, and vomiting), and blood chemistry and serological tests, including total bilirubin (TB), alanine transaminase (ALT), gamma glutamyltransferase (GGT), carbohydrate antigen 19-9 (CA 19-9), carcinoembryonic antigen (CEA), cancer antigen 125 (CA-125), alpha fetoprotein (AFP), and sIgG4, were collected retrospectively. In addition, information on biliary drainage procedures, types of resection, postoperative outcomes, and complications [histopathology, incidence rate, ClavienDindo classification of complications and mortality $(6,7)$ ] were recorded. Patients were divided into the PHC group and the IgG4-SC group, and their clinical and laboratory characteristics were analyzed.

\section{Histopathological evaluation}

The resected specimens of all patients with benign diseases were reevaluated by a professional pathologist who was not informed of the diagnostic information, and the paraffinembedded resected specimens were assessed according to the ICPD (5). The 3 major histopathological features associated with IgG4-SC were dense lymphoplasmacytic infiltration, fibrosis, and focal and occlusive phlebitis. Secondary criteria were phlebitis without cavity occlusion and eosinophilia. The criteria were positive or negative. Immunohistochemical staining of IgG4 plasma cells was also performed, and IgG4 cell counts were obtained for surgical specimens. The diagnostic accuracy of plasma cell numbers depends largely on the selected reference values, and the different organizational conditions and changes. In a case-control study on endoscopic biopsies of the ampulla of Vater and bile duct wall, a threshold of 10 IgG4+ plasma cells/(high power field, HPF) yielded limited sensitivity but reasonable specificity (8). In this study, 2 or 3 histological features combined with more than 10 IgG4 plasma cells/ HPF in each surgical specimen were considered to be highly suggestive of IgG4-SC. Benign disease that did not meet the IgG4-SC histological criteria or other diagnoses were considered atypical inflammation/fibrosis. This study did not reassess and excluded resected specimens from patients with malignant disease.

All procedures performed in this study involving human participants were in accordance with the Declaration of Helsinki (as revised in 2013). This retrospective study was approved by the Ethics Committee of West China Hospital of Sichuan University (2020 Year of Approval No. 388) and waived the need for informed consent.

\section{Statistical analysis}

The characteristics of the different patients in the IgG4SC group and the PHC group were analyzed by descriptive statistical methods, and the differences between groups were compared using the independent sample $t$-test and chi square test. SPSS 26 software was used for statistical analysis, and significant differences were defined as $\mathrm{P}$ value $<0.05$. 
Table 1 Histological diagnosis of the study cohort

\begin{tabular}{ll}
\hline Histological diagnosis & $\mathrm{N}=289$ \\
\hline Perihilar cholangiocarcinoma & $235(81 \%)$ \\
Other malignancy & $28(10 \%)$ \\
Gall bladder carcinoma & 3 \\
$\begin{array}{l}\text { Congenital common bile duct cyst with } \\
\text { malignant transformation }\end{array}$ & 2 \\
$\begin{array}{l}\text { Hepatocellular carcinoma involves hilar bile } \\
\text { ducts }\end{array}$ & 15 \\
Intrahepatic cholangiocarcinoma & 8 \\
Benign disease & $26(9 \%)$ \\
Sclerosing inflammation & 23 \\
Bile duct cystadenomas & 1 \\
Xanthogranulomatous cholangitis & 2 \\
\hline
\end{tabular}

\section{Results}

\section{Population characteristics}

A total of 289 patients underwent radical resection for presumed PHC at our hospital from January 2010 to December 2019. Among the 263 (91\%) patients with malignant disease, 235 (81\%) were diagnosed with PHC and $28(10 \%)$ of them had a malignancy other than PHC, including intrahepatic cholangiocarcinoma, gallbladder carcinoma, and hepatocellular carcinoma involving the perihilar bile ducts.

A total of 26 patients (9\%) had benign disease, without histological evidence of malignancy. Among them, 23 had sclerosing inflammation, 1 had cystadenoma, and 2 had xanthogranulomatous cholangitis. Additionally, 18 had a definitive diagnosis of IgG4-SC. The misdiagnosis rate was $19 \%$ (54/289), of which, 26 patients had benign disease, and 28 patients had malignancies (Table 1).

\section{Histopathological evaluation of IgG4-SC}

A total of 26 resection specimens were available for reevaluation of IgG4-SC, including 23 patients with a sclerosing type of inflammation. In addition, 18 resection specimens met the adjusted pathological criteria for IgG4SC (Figure 1A,B). Moreover, 7 patients scored over 50 $\mathrm{IgG} 4^{+}$plasma cells/HPF, and 22 scored over $10 \mathrm{IgG} 4^{+}$ plasma cells/HPF (Figure 1C, Table 2). The remaining 5 patients were difficult to classify and were excluded from this study.

\section{Demographic, clinical characteristics, blood chemistry, and serology}

Demographic, clinical characteristics, blood chemistry, and serology are shown in Table 3. The mean age of onset was similar between the two groups (59 vs. $57, \mathrm{P}=0.39$ ), however, the percentage of male patients in the IgG4-SC group was significantly higher than in the $\mathrm{PHC}$ group (83\% vs. $59 \%, \mathrm{P}=0.004)$. Compared with $\mathrm{PHC}$ patients, IgG4$\mathrm{SC}$ patients were more prone to abdominal pain and weight loss $(\mathrm{P}<0.001)$.

There was no significant difference in the rate of jaundice between 2 groups ( $76 \%$ vs. $78 \%, \mathrm{P}=0.85$ ), however, patients with $\mathrm{PHC}$ were supported by a higher preoperative TB level (199 vs. $47 \mu \mathrm{mol} / \mathrm{L}, \mathrm{p}<0.001$ ). Furthermore, preoperative ALT and GGT were also significantly higher in the PHC group (143 vs. 73 U/L, $\mathrm{P}=0.013,677$ vs. $391 \mathrm{U} / \mathrm{L}$, respectively, $\mathrm{P}=0.029)$. CA $19-9$ and $\mathrm{CA}-125$ levels were higher in PHC patients compared to IgG4-SC patients (387 vs. 66,59 vs. $26 \mathrm{U} / \mathrm{mL}$, respectively, $\mathrm{P}<0.001$ ), while the two groups had similar CEA levels (16 vs. $9 \mathrm{ng} / \mathrm{mL}, \mathrm{P}=0.73$ ).

\section{Pre-operative drainage, resection type, morbidity, and perioperative mortality}

At the same time as radical resection, patients with PHC received extended hemihepatectomy more often than patients with IgG4-SC ( $88 \%$ vs. $44 \%, \mathrm{P}<0.001)$, and patients with $\mathrm{PHC}$ had more frequent preoperative drainage $(37 \% v s$. $11 \%, \mathrm{P}=0.027)$. However, there was no significant difference in postoperative morbidity between the two groups ( $\mathrm{P}=0.29$; Table 4). The PHC group had higher perioperative mortality, but the difference between the two groups was not statistically significant (3.8\% vs. $0 \%, \mathrm{P}=0.447$; Table 4).

\section{Imaging and diagnostic endoscopic techniques}

This study also evaluated the presence of extrabiliary IgG4-related disease (RD) in patients with IgG4-SC on preoperative MRI/CT scans. Two patients presented with chronic pancreatitis (parenchymal atrophy and calcification), with no typical change in autoimmune pancreatitis (AIP; diffuse enlargement of the pancreas; Figure $2 A, B$ ). No other possible manifestations of IgG4-RD were found in 

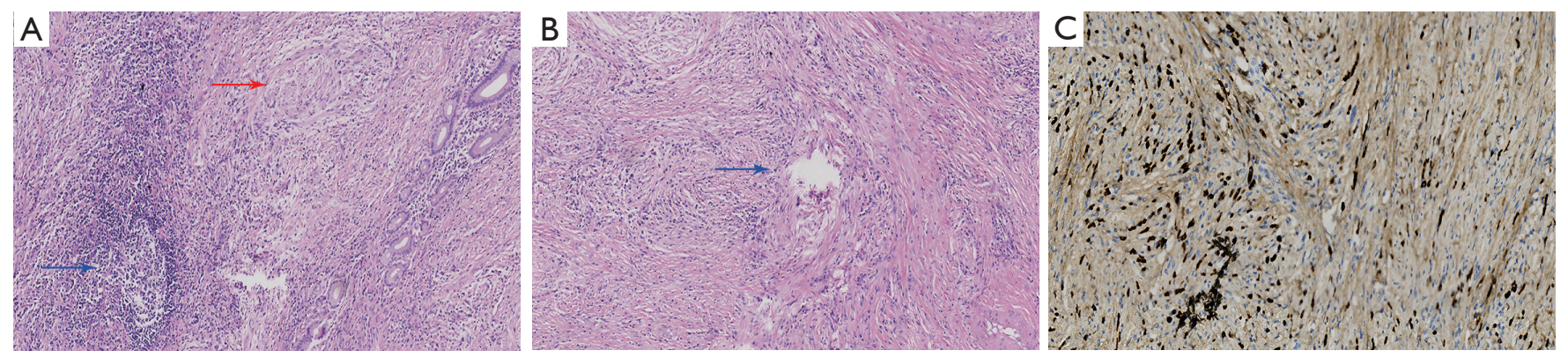

Figure 1 Typical histopathological features of IgG4-SC on resection specimen. (A) A low-magnification microscopic section reveals a prominent lymphoplasmacytic infiltrate (the blue arrow) associated with dense storiform fibrosis (the red arrow) (H\&E, original magnification $\times 200$ ); (B) high-magnification view of the mixed inflammatory infiltrate, diffuse fibrosis and obliterative phlebitis. The blue arrow points out the obliterative phlebitis $(\mathrm{H} \& \mathrm{E}$, original magnification $\times 200)$; (C) the IgG4 immunohistochemical staining highlights the marked infiltrate of IgG4-positive plasma cells (IHC, original magnification $\times 400$ ).

Table 2 Features of IgG-SC on histopathology

\begin{tabular}{lc}
\hline Features of IgG4-SC on histopathology & $\mathrm{n}=23[\%]$ \\
\hline Major histological criteria & $23[100]$ \\
Lymphoplasmacytic infiltration & $18[83]$ \\
Storiform fibrosis & $11[48]$ \\
Obliterative phlebitis & \\
Minor histological criteria & $20[87]$ \\
Phlebitis without obliteration of the lumen & $18[78]$ \\
Eosinophilia & \\
IgG4+ plasma cells infiltration & $22[96]$ \\
$>10$ IgG4+ cells/HPF & $7[30]$ \\
$>50$ IgG4+ cells/HPF & \\
Meeting IgG-SC criteria & $18[78]$ \\
2 of 3 major criteria \& $>10$ IgG4+ cells/HPF & $7[30]$ \\
2 of 3 major criteria \& $>50$ IgG4+ cells/HPF
\end{tabular}

IgG4-SC, immunoglobulin (Ig) G4-related sclerosing cholangitis; HPF, high power field.

preoperative imaging.

In summary, of the 289 patients who underwent $\mathrm{PHC}$ resection, 26 (9\%) were eventually diagnosed as benign. There were 18 patients ( $6 \%$ of the total) diagnosed with IgG4-SC by histology, accounting for $69 \%$ of all benign diseases.

\section{Discussion}

IgG4-SC is a biliary manifestation of IgG4-RD and a subgroup of SC, which is defined as the progressive narrowing and destruction of the bile duct caused by diffuse inflammation and fibrosis. The clinical features of IgG4-SC are: (I) chronic cholestasis enzyme elevation, (II) continuously increasing serum IgG4 levels, (III) intrahepatic and/or extrahepatic bile duct segmental or diffuse stenosis, and bile duct wall thickening, (IV) histologically visible lymphoplasmacytic and IgG4-positive plasma cell infiltration and fibrosis, (V) IgG4-RD in other organs, mainly involving AIP, (VI) effectiveness of steroid therapy.

Unfortunately, due to the rare occurrence of the disease and the lack of a diagnostic gold standard, the clinical, serological, and imaging characteristics of IgG4-SC have not been clearly described. In 2008, Ghazale et al. described the clinical characteristics, treatment responses, and recurrence predictors of 53 IgG4-SC patients (3). Six years later, Huggett et al. found that AIP and IgG4-SC were significantly related to morbidity and mortality in a cohort of 115 patients, including $68 \mathrm{IgG4}$-SC patients, which is the largest series of IgG4-SC cases reported so far (9). At the same time, isolated IgG4-SC without evidence of pancreas or other organ involvement is uncommon, and it is difficult to distinguish it from preoperative $\mathrm{PHC}$.

In this study, we reported the incidence of IgG4-SC in a large cohort of 289 patients resected for presumed primary liver cancer over the past 10 years. We found that 26 patients (9\%) had benign diseases rather than PHC, which was lower than the literature since $2008(10,11)$. This may be due to the improvements in imaging methods over the past decade. Eighteen patients with benign diseases were clearly diagnosed as IgG4-SC. In our study, this disease mainly affected men aged 26 to 72 (male to female ratio 
Table 3 Demographical, clinical characteristics, blood chemistry and serology

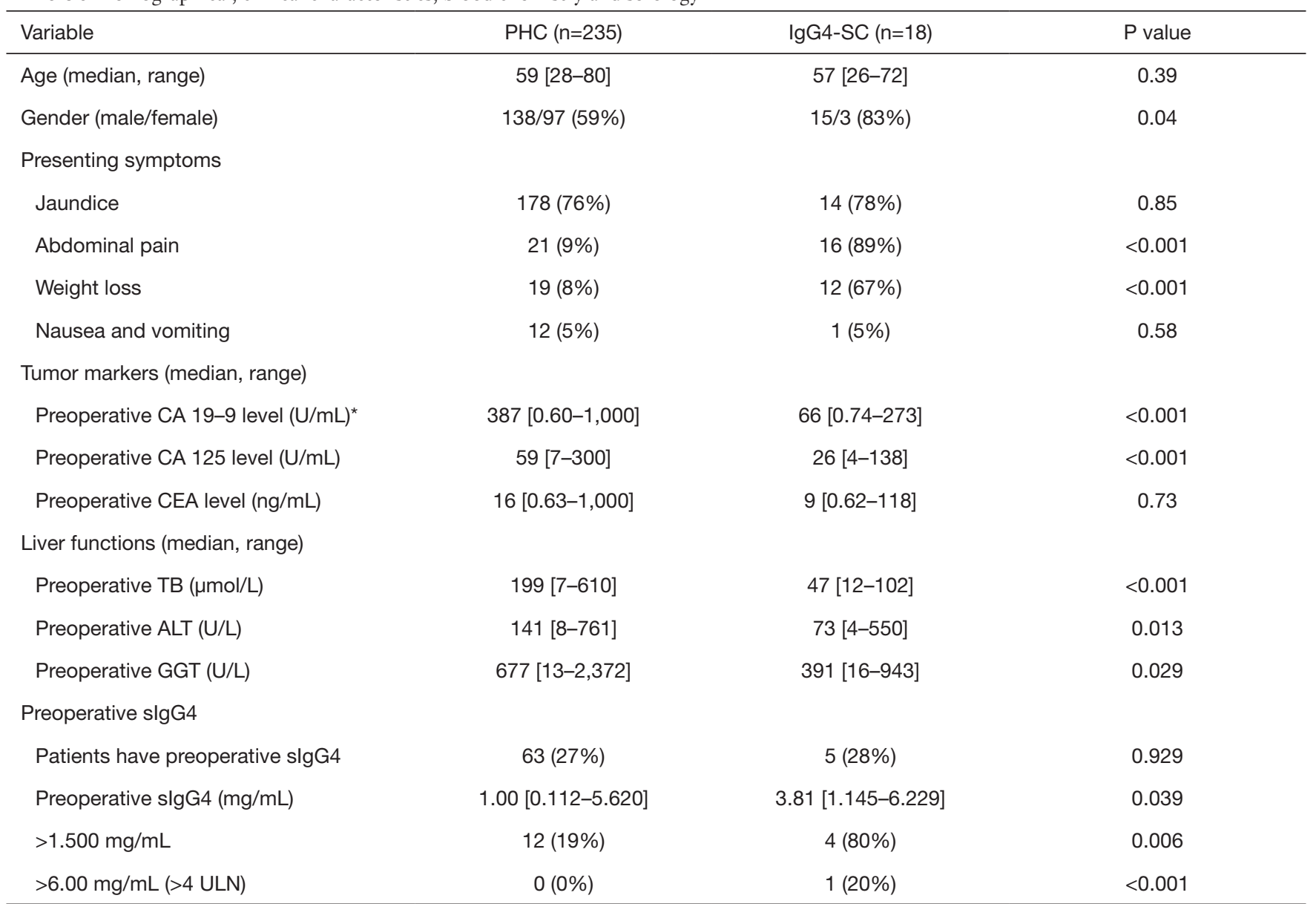

${ }^{\star}$ The maximum limit that our hospital can detect is 1,000 U/mL. PHC, perihilar cholangiocarcinoma; CA 19-9, carbohydrate antigen 19-9; CA 125, carbohydrate antigen 125; CEA, carcinoembryonic antigen; TB, total bilirubin; ALT, alanine transaminase; GGT, gamma glutamyltransferase; slgG4, serum immunoglobulin (lg) G4; ULN, limit of normal.

was 5:1), and it usually manifested as obstructive jaundice, weight loss, and abdominal pain. The preoperative TB, ALT, GGT, CA 19-9, and CA-125 levels in the PHC group were significantly increased, and the incidence of large-area resection in the $\mathrm{PHC}$ group was also higher than that of the IgG4-SC group, suggesting that IgG4-SC mostly showed mild bile duct obstruction and with less cholestasis. Patients with less cholestasis and significantly elevated levels of CA 19-9 and CA-125 were more likely to develop PHC.

Although sIgG4 levels are often elevated in IgG4$\mathrm{SC}$, when they are only slightly elevated $[<4 \times$ upper limit of normal (ULN)] the diagnostic value is limited, as approximately $15 \%$ of $\mathrm{PHC}$ patients have elevated sIgG4 levels, and $20 \%$ of IgG4-SC patients have normal sIgG4 levels. Therefore, merely elevated sIgG4 does not exclude PHC, and using a 2-fold cut-off for sIgG4 may not be able to reliably distinguish IgG4-SC. When the cut-off value is 4 times, ULN-sIgG4 is $100 \%$ specific for IgG4-SC (12). In our study, less than one-third of patients had preoperative sIgG4 levels. The preoperative sIgG4 level of the IgG4-SC group was significantly higher than that of the PHC group ( 3.81 vs. $1.00 \mathrm{mg} / \mathrm{mL}, \mathrm{P}=0.039$ ). Among them, $19 \%$ of PHC patients had elevated sIgG4 levels $(>1.5 \mathrm{mg} / \mathrm{mL})$, and $20 \%$ of IgG4-SC patients had normal sIgG4 levels. The sensitivity and specificity of sIgG4 for diagnosing IgG4-SC were $80 \%$ and $81 \%$, respectively. Therefore, when there is only a slight increase in sIgG4 (1-4× ULN), the diagnostic value is limited. In addition, when the cut-off value is 4 times the ULN, sIgG4 has $100 \%$ specificity for IgG4-SC, however, the sensitivity quickly drops to $20 \%$.

Considering the small sample sizes of the 2 groups, these data may not reflect the true situation. However, 
Table 4 Drainage pre-operation, resection type, morbidity and perioperative mortality

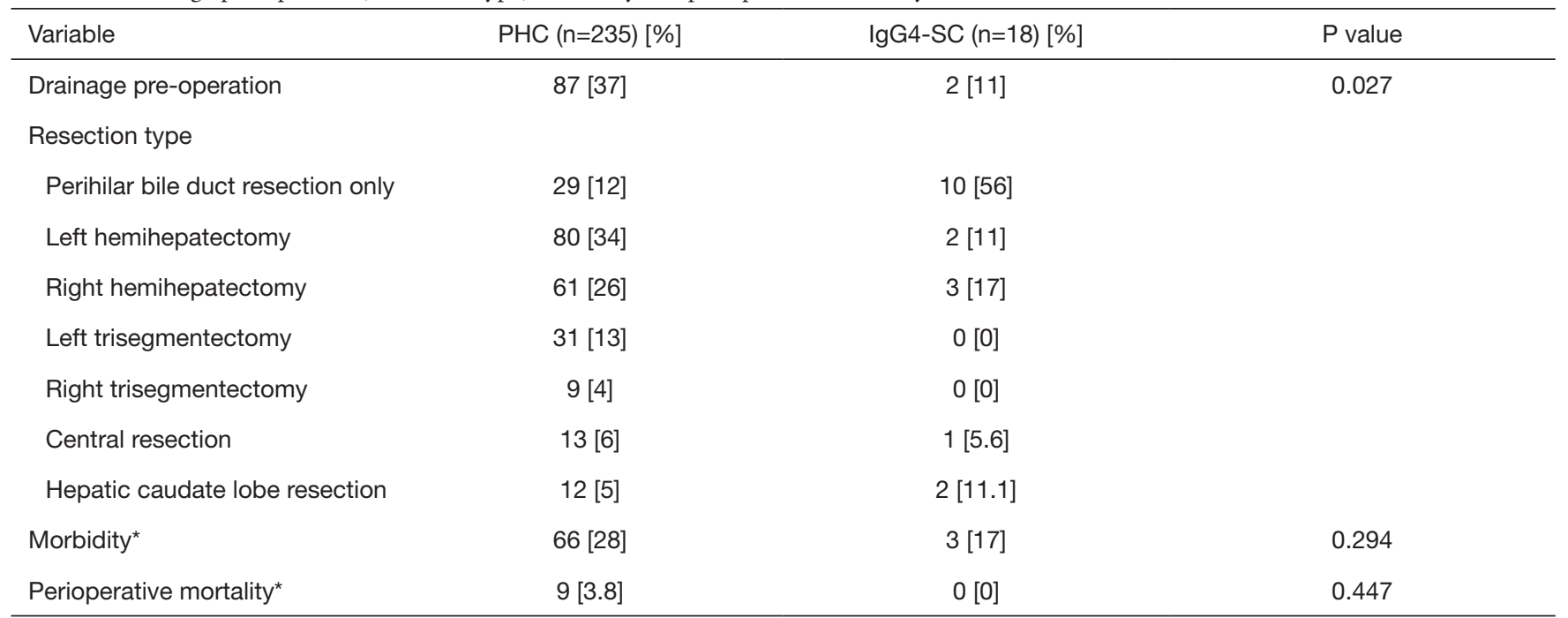

*Part of data were missing due to incomplete records. PHC, perihilar cholangiocarcinoma; IgG4-SC, immunoglobulin (Ig) G4-related sclerosing cholangitis.
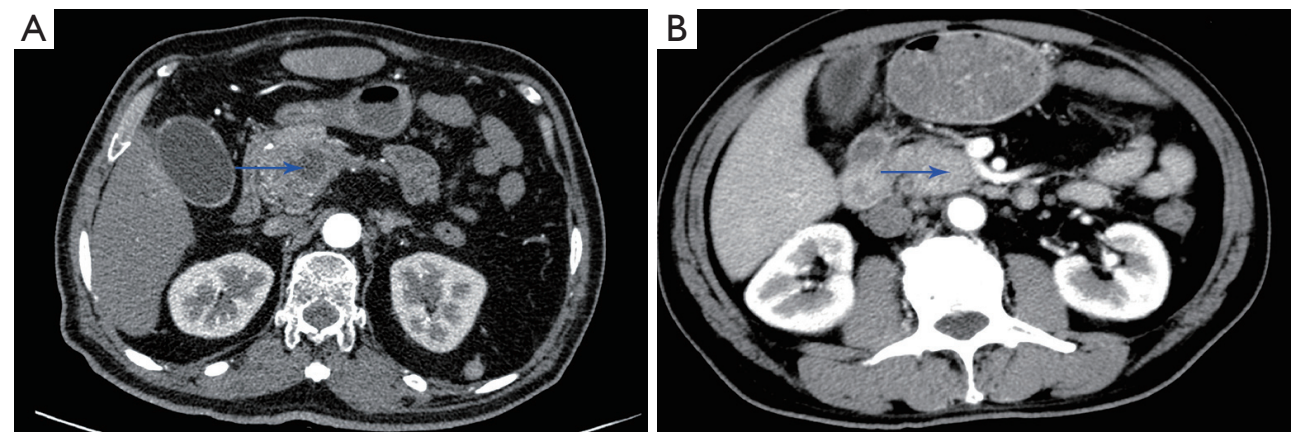

Figure 2 Typical imaging features of chronic pancreatitis. (A) Typical features of chronic pancreatitis, parenchymal atrophy and calcifications, head of the pancreas was enlarged, and the pancreatic tail was slightly shrunk (the blue arrow); (B) the pancreatic parenchyma is atrophied and calcified, head of pancreatic is enlarged with dilated bile ducts (the blue arrow).

increasing the detection rate of $\mathrm{sIgG} 4$ can reduce the misdiagnosis rate to a certain extent. According to research by the National People's Congress, in the background of dense lymphoplasma cell infiltration, IgG4+ plasma cell infiltration, as well as the presence of storage fibrosis and/or phlebitis obliterans, are highly suggestive of IgG4-RD (5). In addition, Masaki et al. also showed that reducing the critical level of $>50 \mathrm{IgG} 4+$ plasma cells/HPF to the critical level of $>10$ IgG4+ plasma cells/HPF, when combined with the above major histological criteria, will not reduce specificity but will increase sensitivity (13). In our study, 2 or 3 histological features combined with $>10$ IgG4+ plasma cells/HPF in each surgical specimen were considered to be highly suggestive of IgG4-SC. By scoring the IgG4+ plasma cell infiltration and typical histopathological characteristics in the excised specimens, we found that $78 \%(n=18 / 23)$ of patients had histological evidence of IgG4-SC, and there were only 7 patients who scored greater than $50 \mathrm{IgG} 4+$ cells in the 23 cases of benign diseases. Furthermore, there were 18 cases of IgG4+ cells/ HPF $>10$ who also met the main histological criteria, which is consistent with the above conclusions.

Although endoscopic biopsy through the bile duct and Vater's nipple is an important method to exclude PHC, it is very difficult to obtain a large enough pathological sample with IgG4-SC characteristics (14). The specimens 
are usually too small to observe the full spectrum of lymphoplasmacytic cholangitis. Therefore, preoperative imaging should be given priority. Cholangiography [ERCP or magnetic resonance cholangiopancreatography (MRCP)] and computed tomography (CT) plus venography can detect stenosis of the bile duct tree, thickening and enhancement of the bile duct wall, dilation of the upstream bile duct, and in some cases soft tissue masses are suspected of being malignant (14-16). By reviewing the imaging data, we found that there were no clinical or imaging features that could clearly distinguish PHC from IgG4-SC. However, the preoperative imaging examinations should always be strict.

In recent years, the awareness of IgG4-SC in patients with primary liver cancer has gradually increased. However, our research results showed that in the past 10 years, the proportion of suspected primary liver cancer patients with IgG4-SC after resection has not significantly decreased. This may be largely due to the lack of an accurate IgG4-SC diagnostic test. A more accurate, non-invasive diagnostic test would be beneficial for patients with malignancies and/ or IgG4-SC. Doorenspleet et al. demonstrated that IgG4/ IgG RNA determined by quantitative polymerase chain reaction (qPCR) has a higher diagnostic accuracy than sIgG4 for IgG4-SC (17). Therefore, it is recommended to include this in the diagnosis of hypothetical PHC patients (18). However, due to the complexity of detection, clinical application takes time.

In addition, diagnostic endoscopy techniques such as choledochoscopy (19-22) and endoscopic ultrasound (EUS) enhance the accuracy of bile duct imaging and tissue sampling, and are in line with current gold standards (such as ERCP-guided brush cytology). Compared with the diagnosis of bile duct stricture, the sensitivity and specificity are higher (23). Intraductal ultrasound (IDUS) can effectively distinguish IgG4-SC and PHC (14). IDUS can obtain high-resolution images of the layered structure of the bile duct wall, and can clearly distinguish the pathological differences between IgG4-SC and PHC. The most valuable characteristic for diagnosing bile duct stricture is the uniform thickening of the inner and outer walls. CT and MRCP can also be used to detect the thickness of the bile duct wall, however, EUS or IDUS can show these subtle changes more clearly. The combined application of MRCP and EUS is therefore helpful for the differential diagnosis of IgG4-SC and PHC.

In our study, only a few patients had IDUS and EUS before surgery, but most patients underwent CT and MRCP. However, no clinical or radiographic features were found that could clearly differentiate IgG4-SC from $\mathrm{PHC}$ and thus prevent a number of unnecessary resections in patients with IgG4-SC. Two patients with IgG4$\mathrm{RD}$ had signs of chronic pancreatitis on initial imaging examinations, but were not typical signs of AIP. Even so, preoperative imaging and endoscopic diagnostic techniques should always strictly examine the signs of extrabiliary manifestations of IgG4-RD.

In conclusion, it is difficult to distinguish IgG-SC from PHC. In the past 10 years, this misdiagnosis has resulted in a large number of ineffective hepatectomies. Improving the detection rate of sIgG4 may therefore avoid misdiagnosis, surgery, and life-threatening complications. Furthermore, when the cut-off value is 4 times the ULN, sIgG4 may strongly indicate IgG4-SC. For patients who intend to receive surgical treatment, preoperative IDUs and EUS examinations are recommended.

\section{Acknowledgments}

Funding: This study was supported by grants from the Key Technology Research and Development Program of Sichuan Province (2018SZ0225).

\section{Footnote}

Reporting Checklist: The authors have completed the STROBE reporting checklist. Available at http://dx.doi. org/10.21037/atm-21-140

Data Sharing Statement: Available at http://dx.doi. org/10.21037/atm-21-140

Conflicts of Interest: All authors have completed the ICMJE uniform disclosure form (available at http://dx.doi. org/10.21037/atm-21-140). The authors have no conflicts of interest to declare.

Ethical Statement: The authors are accountable for all aspects of the work in ensuring that questions related to the accuracy or integrity of any part of the work are appropriately investigated and resolved. All procedures performed in this study involving human participants were in accordance with the Declaration of Helsinki (as revised in 2013). This retrospective study was approved by the Ethics Committee of West China Hospital of Sichuan University (2020 Year of Approval No. 388) and waived the need for informed consent. 
Open Access Statement: This is an Open Access article distributed in accordance with the Creative Commons Attribution-NonCommercial-NoDerivs 4.0 International License (CC BY-NC-ND 4.0), which permits the noncommercial replication and distribution of the article with the strict proviso that no changes or edits are made and the original work is properly cited (including links to both the formal publication through the relevant DOI and the license). See: https://creativecommons.org/licenses/by-nc-nd/4.0/.

\section{References}

1. Hubers LM, Maillette de Buy Wenniger LJ, Doorenspleet ME, et al. IgG4-Associated Cholangitis: A Comprehensive Review. Clin Rev Allergy Immunol 2015;48:198-206.

2. Stone JH, Zen Y, Deshpande V. IgG4-related disease. N Engl J Med 2012;366:539-51.

3. Ghazale A, Chari ST, Zhang L, et al. Immunoglobulin G4-associated cholangitis: clinical profile and response to therapy. Gastroenterology 2008;134:706-15.

4. Erdogan D, Kloek JJ, ten Kate FJ, et al. Immunoglobulin G4-related sclerosing cholangitis in patients resected for presumed malignant bile duct strictures. Br J Surg 2008;95:727-34.

5. Deshpande V, Zen Y, Chan JK, et al. Consensus statement on the pathology of IgG4-related disease. Mod Pathol 2012;25:1181-92.

6. Clavien PA, Barkun J, de Oliveira ML, et al. The ClavienDindo classification of surgical complications: five-year experience. Ann Surg 2009;250:187-96.

7. Dindo D, Demartines N, Clavien PA. Classification of surgical complications: a new proposal with evaluation in a cohort of 6336 patients and results of a survey. Ann Surg 2004;240:205-13.

8. Kawakami H, Zen Y, Kuwatani M, et al. IgG4-related sclerosing cholangitis and autoimmune pancreatitis: histological assessment of biopsies from Vater's ampulla and the bile duct. J Gastroenterol Hepatol 2010;25:1648-55.

9. Huggett MT, Culver EL, Kumar M, et al. Type 1 autoimmune pancreatitis and IgG4-related sclerosing cholangitis is associated with extrapancreatic organ failure, malignancy, and mortality in a prospective UK cohort. Am J Gastroenterol 2014;109:1675-83.

10. Kloek JJ, van Delden OM, Erdogan D, et al. Differentiation of malignant and benign proximal bile duct strictures: the diagnostic dilemma. World J Gastroenterol
2008;14:5032-8.

11. Gerhards MF, Vos P, van Gulik TM, et al. Incidence of benign lesions in patients resected for suspicious hilar obstruction. Br J Surg 2001;88:48-51.

12. Oseini AM, Chaiteerakij R, Shire AM, et al. Utility of serum immunoglobulin G4 in distinguishing immunoglobulin G4-associated cholangitis from cholangiocarcinoma. Hepatology 2011;54:940-8.

13. Masaki Y, Kurose N, Yamamoto M, et al. Cutoff Values of Serum IgG4 and Histopathological IgG4+ Plasma Cells for Diagnosis of Patients with IgG4-Related Disease. Int J Rheumatol 2012;2012:580814.

14. Naitoh I, Nakazawa T, Ohara H, et al. Endoscopic transpapillary intraductal ultrasonography and biopsy in the diagnosis of IgG4-related sclerosing cholangitis. J Gastroenterol 2009;44:1147-55.

15. Tokala A, Khalili K, Menezes R, et al. Comparative MRI analysis of morphologic patterns of bile duct disease in IgG4-related systemic disease versus primary sclerosing cholangitis. AJR Am J Roentgenol 2014;202:536-43.

16. Vlachou PA, Khalili K, Jang HJ, et al. IgG4related sclerosing disease: autoimmune pancreatitis and extrapancreatic manifestations. Radiographics 2011;31:1379-402.

17. Doorenspleet ME, Hubers LM, Culver EL, et al. Immunoglobulin G4(+) B-cell receptor clones distinguish immunoglobulin G 4-related disease from primary sclerosing cholangitis and biliary/pancreatic malignancies. Hepatology 2016;64:501-7.

18. Tabibian JH, Lindor KD. Distinguishing immunoglobulin G4-related disease from its pancreatobiliary mimics: Are we there now? Hepatology 2016;64:340-3.

19. Fukuda $Y$, Tsuyuguchi T, Sakai $Y$, et al. Diagnostic utility of peroral cholangioscopy for various bile-duct lesions. Gastrointest Endosc 2005;62:374-82.

20. Kurihara T, Yasuda I, Isayama H, et al. Diagnostic and therapeutic single-operator cholangiopancreatoscopy in biliopancreatic diseases: Prospective multicenter study in Japan. World J Gastroenterol 2016;22:1891-901.

21. Tsuyuguchi T, Fukuda Y, Saisho H. Peroral cholangioscopy for the diagnosis and treatment of biliary diseases. $\mathrm{J}$ Hepatobiliary Pancreat Surg 2006;13:94-9.

22. Navaneethan U, Njei B, Venkatesh PG, et al. Endoscopic ultrasound in the diagnosis of cholangiocarcinoma as the etiology of biliary strictures: a systematic review and meta-analysis. Gastroenterol 
Rep (Oxf) 2015;3:209-15.

23. Navaneethan U, Njei B, Lourdusamy V, et al. Comparative effectiveness of biliary brush cytology and intraductal biopsy for detection of malignant biliary strictures: a systematic review and meta-analysis. Gastrointest Endosc 2015;81:168-76.

(English Language Editor: C. Betlazar-Maseh)

Cite this article as: Feng L, You Z, Ma D, Yan L, Cheng H, Gou J, Chen L. Immunoglobulin (Ig) G4-related sclerosing cholangitis in patients resected for presumed perihilar cholangiocarcinoma: a 10-year experience. Ann Transl Med 2021;9(5):415. doi: 10.21037/atm-21-140 